INPLASY

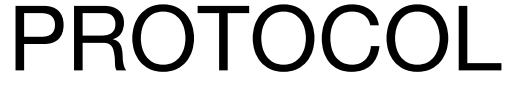

To cite: Zhai et al. A meta analysis of PD-1 agents in ESSCLC. Inplasy protocol 202130035. doi:

10.37766/inplasy2021.3.0035

Received: 11 March 2021

Published: 12 March 2021

Corresponding author:

Linzhu Zhai

lizhulin@hotmail.com

Author Affiliation:

the First Affiliated Hospital of Guangzhou University of Chinese Medicine

Support: National Natural Science Found.

Review Stage at time of this submission: Data extraction.

Conflicts of interest:

None declared.

\section{A meta analysis of PD-1 agents in ES-SCLC}

Review question / Objective: We conducted a meta-analysis on the efficacy and safety of PD-1 agents in first-line and maintenance treatment for extensive-stage small-cell lung cancer.

Condition being studied: PD-1 agents in ES-SCLC. We had finished the study selection and data extraction.

Information sources: The studies were searched from Pubmed, Embase and ClinicalTrials.gov databases up to $\mathbf{3 0}$ December 2020 using a combination of the main search terms "SCLC or small-cell lung cancer" and "nivolumab or pembrolizumab" within the restriction limit of "clinical trial". We also included complete and updated outcomes on important international conferences (WCLC, ASCO, ESMO, CSCO) by inspecting relevant ppt files.

INPLASY registration number: This protocol was registered with the International Platform of Registered Systematic Review and Meta-Analysis Protocols (INPLASY) on 12 March 2021 and was last updated on 12 March 2021 (registration number INPLASY202130035).

\section{INTRODUCTION}

Review question / Objective: We conducted a meta-analysis on the efficacy and safety of PD-1 agents in first-line and maintenance treatment for extensive-stage small-cell lung cancer.
Condition being studied: PD-1 agents in ES-SCLC. We had finished the study selection and data extraction.

\section{METHODS}

Participant or population: Patients with histologically or cytologically confirmed ES-SCLC. A total of 4 randomized trials and 
1566 patients met the inclusion criteria and were included in this review and analysis.

Intervention: PD-1 agents.

Comparator: Therapy without PD-1 agents.

Study designs to be included: Clinical trials comparing PD-1 drugs therapy with no PD-1 drugs therapy.

Eligibility criteria: Trials that belonged to phase II/III randomized controlled trials;Trials that enrolled patients with histologically or cytologically confirmed ES-SCLC; Trials that compared any two or more different arms; Trials that reported on at least one of the following clinical outcome measures, ORR, PFS, OS, and AEs:

Information sources: The studies were searched from Pubmed, Embase and ClinicalTrials.gov databases up to 30 December 2020 using a combination of the main search terms "SCLC or small-cell lung cancer" and "nivolumab or pembrolizumab" within the restriction limit of "clinical trial". We also included complete and updated outcomes on important international conferences (WCLC, ASCO, ESMO, CSCO) by inspecting relevant ppt files.

Main outcome(s): Four studies were included. Anti-PD-1 drugs combined with chemotherapy had a great beneficial in ORR (HR:1.13;95\% Cl:1.00-1.28; P = 0.04) , and also achieved a significant PFS gain in first-line treatment (HR:0.75;95\% $\mathrm{Cl}: 0.65-0.86 ; \mathrm{P}<0.0001)$ and maintenance treatment (HR:0.69;95\% Cl:0.61-0.79; P < $0.00001)$. The OS advantage was found in first-line treatment (HR: 0.75; 95\% Cl: 0.640.89; $P=0.0006$ ) , but did not found in maintenance therapy(HR: $0.88 ; 95 \% \mathrm{Cl}$ : 0.76-1.01; $P=0.07$ ). Indirect Treatment Comparison analysis Confirm that the nivolumab and pemblizumab were similar in clinical efficacy. Finally, PD-1 agents had good security.
Quality assessment / Risk of bias analysis: We assessed the risk of bias of individual studies using the Cochrane Risk of Bias Tool. Overall, the risk of bias was low, particularly in the random sequence generation and in the reporting of outcomes.

Strategy of data synthesis: All the studies were analyzed by two independent readers with the direction of a predefined protocol. Discrepancies were resolved by discussion with a third reader. The information extracted from articles were as follows: study title, experimental arm, number of patients, objective response rate, median progression-free survival, median overall survival, adverse events of grade 3-4. The principal evaluation criteria were ORR, OS and PFS. Secondary criteria were safety. We preferred the use of treatment-related adverse events, but if not specified as treatment-related, we used all adverse events.

Subgroup analysis: No subgroup analysis.

Sensitivity analysis: Considering the one of the studes was designed to addition PD-1 agents in third chemotherapy cycle, we also conducted a sensitivity analysis in PFS and $O S$ data. The result did not change significantly and suggested that there was no existing heterogeneity.

\section{Country(ies) involved: China.}

Keywords: small-cell lung cancer; antiPD-1 drugs; immunotherapy; metaanalysis.

Contributions of each author:

Author 1 - Linzhu Zhai.

Author 2 - Lizhu Lin.

Author 3 - Jiahao Tao.

Author 4 - Ling Zhou.

Author 5 - Zeyu Liu.

Author 6 - Yanqun Zhou.

Author 7 - Cuifen Zhang. 